\title{
Cytoprotective Effects of Docosyl Cafferate against tBHP- Induced Oxidative Stress in SH-SY5Y Human Neuroblastoma Cells
}

\author{
Yong-Jun Choi ${ }^{1, a}$, Eun-Bee Kwak ${ }^{1, a}$, Jae-Won Lee ${ }^{1}$, Yong-Suk Lee ${ }^{2}$, Il-Young Cheong ${ }^{2}$, Hee Jae Lee', \\ Sung-Soo Kim ${ }^{1}$, Myong-Jo Kim ${ }^{3}$, Yong-Soo Kwon ${ }^{4}$ and Wanjoo Chun ${ }^{1, *}$ \\ Departments of ${ }^{1}$ Pharmacology, ${ }^{2}$ Anesthesiology, College of Medicine, ${ }^{3}$ Division of Bio-resources Technology, \\ ${ }^{4}$ College of Pharmacy, Kangwon National University, Chuncheon 200-701, Republic of Korea
}

\begin{abstract}
Neuronal cell death is a common characteristic feature of a variety of neurodegenerative disorders including Alzheimer's disease and Parkinson's disease. However, there have been no effective drugs to successfully prevent neuronal death in those diseases. In the present study, docosyl cafferate (DC), a derivative of caffeic acid, was isolated from Rhus verniciflua and its protective effects on tBHP-induced neuronal cell death were examined in SH-SY5Y human neuroblastoma cells. Pretreatment of DC significantly attenuated tBHP-induced neuronal cell death in a concentration-dependent manner. DC also significantly suppressed tBHP-induced caspase-3 activation. In addition, DC restored tBHP-induced depletion of intracellular Bcl-2, an anti-apoptotic member of the Bcl-2 family. Furthermore, DC significantly suppressed tBHP-induced degradation of IKB, which retains NF-kB in the cytoplasm, resulting in the suppression of nuclear translocation of NF-kB and its subsequent activation. Taken together, the results clearly demonstrate that DC exerts its neuroprotective activity against tBHP-induced oxidative stress through the suppression of nuclear translocation of NF-kB.
\end{abstract}

Key Words: Docosyl cafferate (DC), SH-SY5Y human neuroblastoma cells, tBHP, Apoptosis, NF-kB

\section{INTRODUCTION}

Oxidative stress has been implicated in diverse neurodegenerative disorders including Alzheimer's disease (AD), Parkinson's disease (PD), and many inflammatory conditions (Simonian and Coyle, 1996; Liu et al., 2002). Many physiological and environmental toxicants exert oxidative stress to cell by overproducing reactive oxygen species (ROS), such superoxide anions and hydroxyl radicals (Halliwell, 1989; Nohl et al., 1996). An increasing evidence suggests that neuronal apoptosis is involved in the pathological processes of neurodegenerative disorders such as AD and PD (Friedlander, 2003). Therefore, novel pharmacological agents that can suppress oxidative stress-induced neuronal damages might provide a promising therapeutic strategy for numerous neuronal deathrelated CNS pathologies.

Rhus verniciflua of the Anacardiaceae family, commonly known as the lacquer tree, has been reported to possess a wide range of biological activities such as antioxidant (Kim et al., 1997; Kim and Choi, 2002), anti-microbial, anti-cancer (Park et al., 2004; Jeong and Park, 2008), and anti-platelet properties (Jeon et al., 2006). However, the exact chemical components of Rhus verniciflua have not been fully elucidated. Recently, it has been reported that stigmasterols isolated from Rhus verniciflua exhibit neuroprotective activity in a mouse model of kainic acid-induced excitotoxicity (Byun et al., 2010).

To further provide biologically active compounds from Rhus verniciflua, docosyl cafferate (DC) was purified from the stem bark of Rhus verniciflua. DC is an ester derivative of caffeic acid that has been reported to possess a wide variety of biological activities such as antioxidant and anti-inflammatory properties (Kim and Kim, 2000) and neuroprotective effect (Sul et al., 2009). Ester derivatives of caffeic acid have also been reported to possess numerous biological activities (Kim, 2010). For example, caffeic acid phenylethyl ester (CAPE) www.biomolther.org

Open Access DOI: 10.4062/biomolther.2011.19.2.195

pISSN: 1976-9148 elSSN: 2005-4483

Copyright $\odot 2011$ The Korean Society of Applied Pharmacology
Received Feb 1, 2011 Revised Mar 3, 2011 Accepted Mar 3, 2011

\section{*Corresponding Author}

E-mail: wchun@kangwon.ac.kr

Tel: +82-33-250-8853, Fax: +82-33-255-8809

${ }^{2}$ These authors contributed equally to this work and shold be considered co-first authors. 
has been reported to possess protective activities against glutamate-induced neurotoxicity (Wei et al., 2008) and cerebral ischemia (Tsai et al., 2006). Although DC was previously identified in the root of Sohora subprostrata (Komatsu et al., 1970), its biological activity has never been demonstrated.

In the present study, we investigated the neuroprotective activity of DC, isolated from the stem bark of Rhus verniciflua, and a possible underlying mechanism by which DC exerts its neuroprotective action in tBHP-induced neuronal cell death in SH-SY5Y human neuroblastoma cells.

\section{MATERIALS AND METHODS}

\section{Chemicals}

Docosyl cafferate (DC, CAS No. 50432-89-8) (Fig. 1) was isolated from Rhus verniciflua and dissolved in $99 \%$ ethanol. Briefly, the dried stem bark of Rhus verniciflua was extracted with methanol and then partitioned with n-hexane, dichlormethane, ethyl acetate, and butanol, successively. DC was isolated from a dichlormethane fraction using silica gel column chromatography. The chemical structure of the compound was determined by means of $1 \mathrm{H}-\mathrm{NMR}(600 \mathrm{MHz})$ and El-MS and identified to $D C$ by comparison with spectral data from the literature (linuma et al., 1992). tert-Butyl hydroperoxide (tBHP, Sigma Chemical Co. MO, USA), a prototypical organic oxidant that has been extensively used in the study of oxidant-induced cell death (Liu et al., 1998; Amoroso et al., 1999), was used to induce oxidative stress in SH-SY5Y cells in the present study.

\section{Cell culture and drug treatment}

Human neuroblastoma SH-SY5Y cells obtained from American Type Culture Collection (Rockville, USA) were cultured in DMEM medium supplemented with $10 \%$ heat-inactivated fetal calf serum, $20 \mathrm{mM}$ glutamine, $10 \mathrm{U} / \mathrm{ml}$ penicillin, and $100 \mu \mathrm{g} /$ $\mathrm{ml}$ streptomycin and maintained at $37^{\circ} \mathrm{C}$ in a humidified atmosphere with $5 \% \mathrm{CO}_{2}$. DC was added to the cells $6 \mathrm{hr}$ prior to the incubation with tBHP for $12 \mathrm{hr}$. All experiments were carried out on sub-confluent cultures.

\section{Determination of cell viability}

SH-SY5Y cells were placed into 12-well culture plates and were incubated at $37^{\circ} \mathrm{C}$ under a $5 \% \mathrm{CO}_{2} / 95 \%$ humidified air incubator for $24 \mathrm{hr}$. Cells were then incubated DC for $6 \mathrm{hr}$ prior to treatment with tBHP for another $12 \mathrm{hr}$. Cell viability was determined using MTT (3-(4,5-dimethylthiazol-2-yl)-2,5-diphenyl

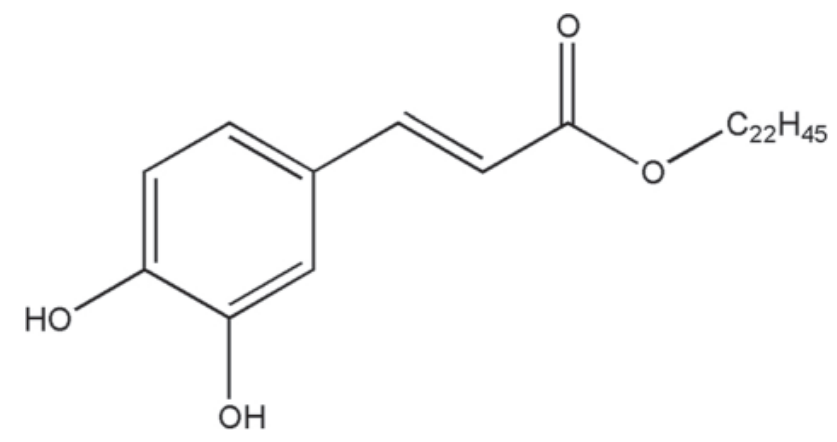

Fig. 1. Chemical structure of docosyl cafferate (DC). tetrazolium bromide) assay, which yields a blue formazan product in living cells, but not in dead cells or their lytic debris. MTT dissolved in phosphate-buffered saline was added at the end of incubation to a final concentration of $0.5 \mathrm{mg} / \mathrm{ml}$, and then incubated at $37^{\circ} \mathrm{C}$ for $2 \mathrm{hr}$, the resultant formazan product was extracted for $4 \mathrm{hr}$ with extraction solution (20\% SDS, $50 \%$ DMF and $2 \%$ acetic acid) and detected by a UV-VIS spectrometer (Perkin Elmer Co.) at $570 \mathrm{~nm}$.

\section{Western blot analysis}

SH-SY5Y cells were washed with PBS two times and lysed with lysis buffer [50 mM Tris- $\mathrm{HCl}, \mathrm{pH} 8.0,150 \mathrm{mM} \mathrm{NaCl}$, $0.02 \%$ sodium azide, $0.1 \%$ sodium dodecyl sulfate (SDS), $1 \%$ NP-40, 0.5\% sodium deoxycholate, $1 \mathrm{mM}$ phenyl methylsulfonyl fluoride]. Equal amounts of protein were separated on $10 \%$ SDS-polyacrylamide gel. Proteins were transferred to Hypond PVDF membrane (Amersham Biosciences, Piscataway, NJ, USA). The membranes were blocked in 5\% skim milk in TBST for $1 \mathrm{hr}$ at room temperature and sequentially incubated with an appropriate antibody; anti-cleaved caspase-3 (Sigma, Saint Louis, MO. USA), anti-cleaved PARP (Sigma), anti-Bcl2 (Sigma), anti-lkB- $\alpha$ (Santa Cruz Biotechnology Inc., Eugene, OR, USA), or $\beta$-actin (Sigma). After thoroughly washing with TBST, the membranes were then washed three times with TBST and incubated with HRP-conjugated goat anti-rabbit IgG (Jackson ImmunoResearch) or HRP-conjugated goat anti-mouse IgG (Jackson ImmunoResearch) for $2 \mathrm{hr}$ at room temperature. The blots were developed by the enhanced chemiluminescence detection kit (ECL Amersham Biosciences, Piscataway, NJ, USA). The immunoblots were quantified by measuring the density of each band using densitometry with ImageQuant ${ }^{\mathrm{TM}}$ software.

\section{Immunocytochemistry}

The effect of DC on the nuclear translocation of $p 65$ subunit of NF-kB was examined by immunofluorescence assay using confocal microscopy. SH-SY5Y cells were cultured in sterile coverslips and pretreated with DC for $1 \mathrm{hr}$ and stimulated with LPS. At 30 min after the tBHP treatment, the cells were fixed in $4 \%$ paraformaldehyde for $20 \mathrm{~min}$ at room temperature. The fixed cells were then permeabilized with $0.1 \%$ Triton X-100 in PBS and blocked with $3 \%$ BSA. Afterwards, the cells were sequentially incubated with rabbit p65 antibody (Santa Cruz Biotechnology Inc., Eugene, OR, USA) at room temperature and Alexa 546-labeled goat anti-rabbit IgG (Molecular Probes, Eugene, OR, USA) at room temperature for $1 \mathrm{hr}$. Nuclei were stained with $0.5 \mu \mathrm{g} / \mathrm{ml}$ of propidium iodide (PI) staining solution for $10 \mathrm{~min}$ at room temperature. After washing with PBS, the sample were mounted and observed by means of confocal microscopy.

\section{Statistical analysis}

All values shown in the figures are expressed as the mean \pm SD obtained from at least three independent experiments. Statistical analysis was carried out by one-way analysis of variance (ANOVA) with Tukey's post-hoc test using SPSS software 12K (SPSS, Chicago, IL, USA). A value of $p<0.05$ was considered as statistically significant. 


\section{RESULTS}

DC attenuates tBHP-induced neuronal cell death in SHSY5Y human neuroblastoma cells

In the present study, neuroprotective effects of DC were examined with tBHP-induced oxidative stress model using SH-SY5Y human neuroblastoma cells. To obtain appropriate concentration of tBHP, which exhibits approximately $50 \%$ of cell death, SH-SY5Y cells were treated with indicated concentrations of tBHP for $12 \mathrm{hr}$, and cell viability was determined using MTT assay. Concentration-dependent cell death was observed with tBHP treatment (Fig. 2A) and $250 \mu \mathrm{M}$ tBHP, which exhibited approximately $50 \%$ cell death, was chosen in the following experiments.

To examine possible protective effects of DC on neuronal cell viability, SH-SY5Y cells were pretreated with DC for $6 \mathrm{hr}$ prior to tBHP treatment. As shown in Fig. 2B, DC significantly attenuated tBHP-induced cell death in a concentration-dependent manner (Fig. 2B). To examine whether tBHP-induced cell death in SH-SY5Y cells was mediated through apoptosis, activation of caspase-3, which is the effector caspase in the apoptotic cascade, was measured. Treatment of the cells with tBHP resulted in activation of casapse- 3 and pretreatment of DC
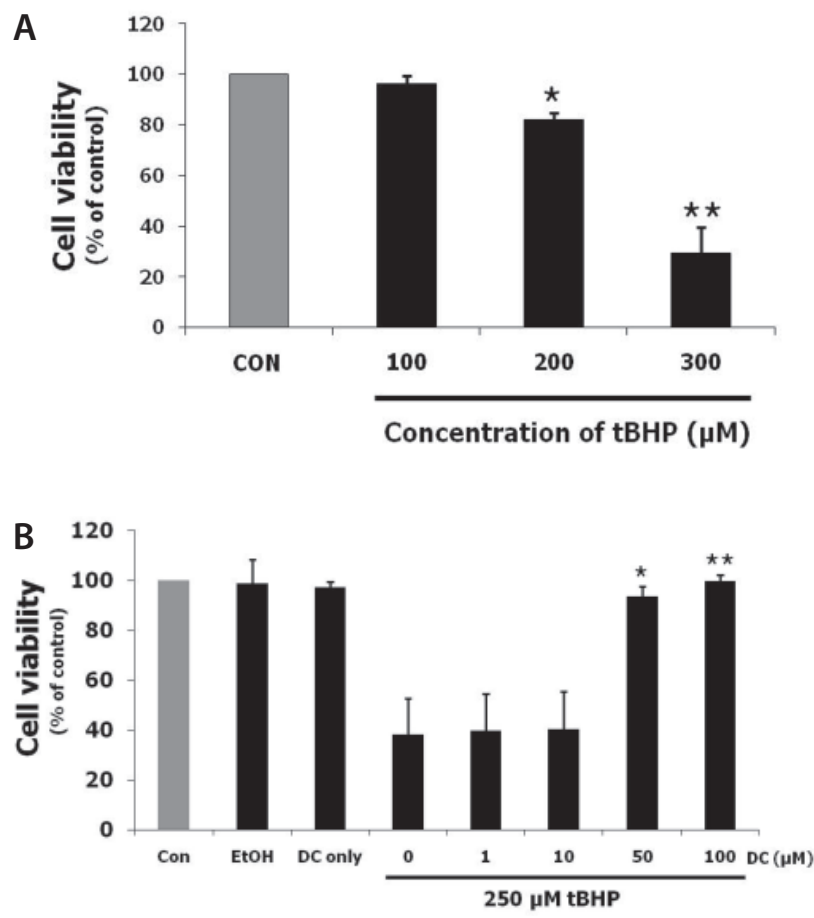

Fig. 2. Concentration-dependent cytotoxicity of $t B H P(A)$ and protective effect of DC (B) on tBHP-induced neuronal cell death in SH-SY5Y human neuroblastoma cells. (A) After $12 \mathrm{hr}$ of incubation with tBHP, neuronal cell viability at indicated concentrations of tBHP was measured using MTT assay. Concentration-dependent cell death was observed and $250 \mu \mathrm{M}$ concentration, which exhibited approximately $50 \%$ cell death, was chosen in the following experiments. (B) tBHP-induced neuronal death was significantly attenuated with $\mathrm{DC}$ treatment in a concentration-dependent manner. No noticeable cell death was observed with DC-only treatment. Data represent three independent experiments and are expressed as mean \pm SD. ${ }^{*} p<0.05$ and ${ }^{* *} p<0.01$ indicate statistically significant difference with tBHP alone. significantly attenuated tBHP-induced caspase-3 activation in a concentration-dependent manner (Fig. 3). These results demonstrate that DC possesses inhibitory effects on tBHPinduced apoptotic cell death in SH-SY5Y cells.

\section{DC inhibits tBHP-induced degradation of Bcl-2 in SH-SY5Y human neuroblastoma cells}

Given the fact that $\mathrm{Bcl}-2$ family members play an important role in regulating cytochrome $c$ release and caspase- 3

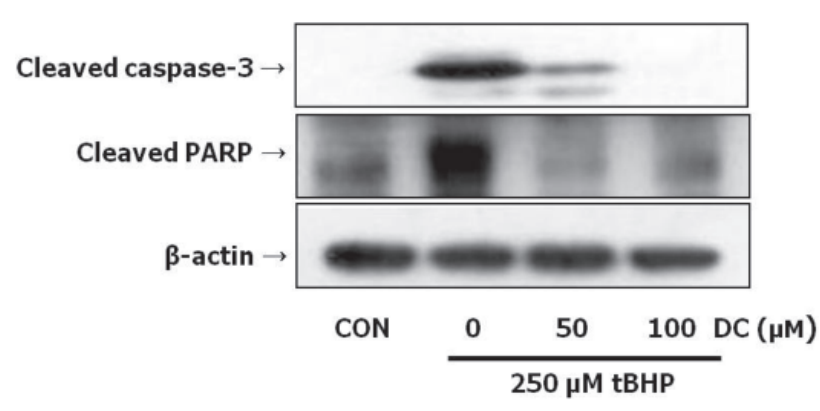

Fig. 3. Attenuation of tBHP-induced apoptosis by DC in SH-SY5Y human neuroblastoma cells. Cells were pretreated with indicated concentration of DC for $6 \mathrm{hr}$ and then incubated with tBHP for 12 hr. Cell lysates were subjected to SDS/PAGE and immunoblotted with caspase-3 or PARP antibodies. Actin antibody was used as a control for equal loading. tBHP-induced apoptotic neuronal death was significantly attenuated with DC treatment in a concentrationdependent manner.

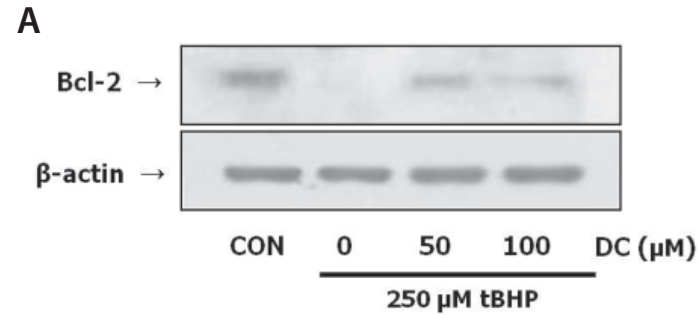

B

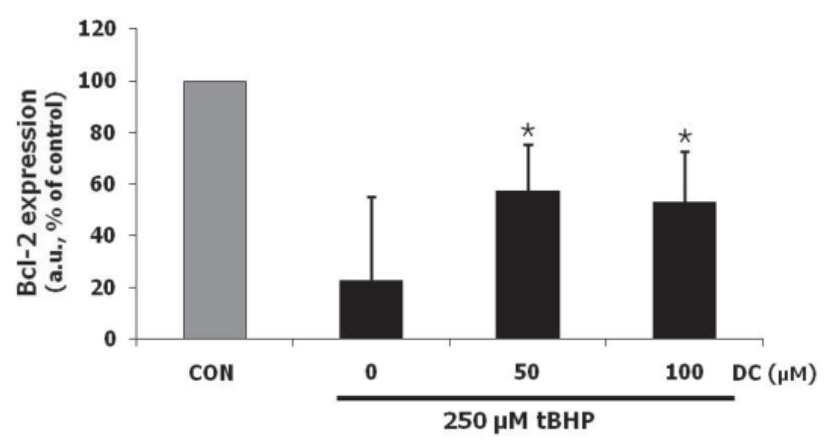

Fig. 4. Effects of DC on the intracellular level of Bcl-2 in tBHPexposed SH-SY5Y human neuroblastoma cells. Intracellular level of $\mathrm{Bcl}-2$ was determined with immunoblotting analysis: a representatative immunoblot $(A)$ and quantitative analysis (B) of immunoblots. $\beta$-Actin was used as an internal control. Quantitative data represent three independent experiments and are expressed as mean $\pm \mathrm{SD}$. ${ }^{*} p<0.05$ indicates statistically significant difference with tBHP alone. 
A

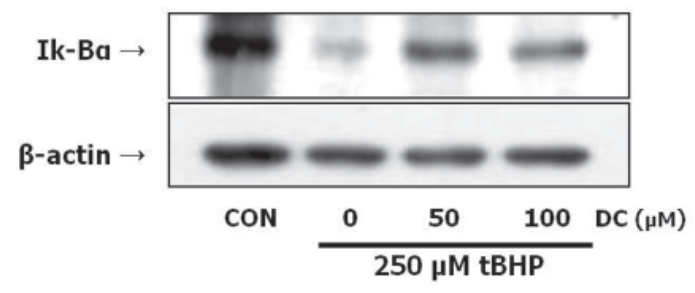

B

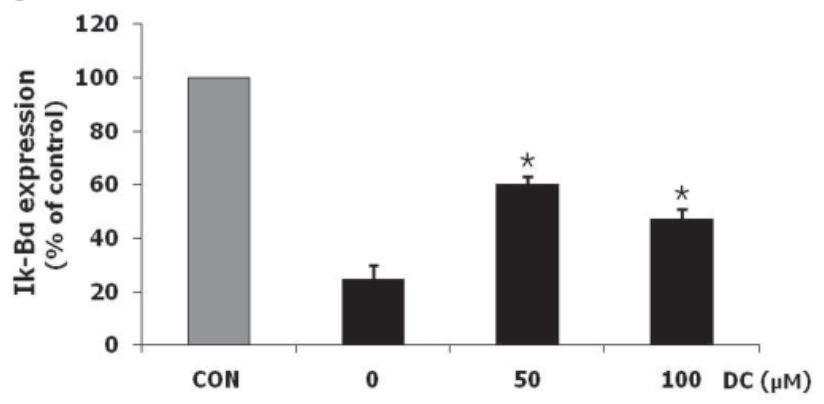

Fig. 5. Inhibitory effects of DC on tBHP-induced IkB- $\alpha$ degradation in SH-SY5Y human neuroblastoma cells. Intracellular level of IKB$\alpha$ was determined with immunoblotting analysis: a representatative immunoblot (A) and quantitative analysis (B) of immunoblots IKB$\alpha$. $\beta$-Actin was used as an internal control. Quantitative data represent three independent experiments and are expressed as mean \pm SD. ${ }^{*} p<0.05$ indicates statistically significant difference with tBHP alone.

activation. The change in intracellular level of $\mathrm{Bcl}-2$, an antiapoptotic protein, was examined upon tBHP stimulation (Fig. 4). Treatment of tBHP resulted in the apparent depletion of intracellular $\mathrm{Bcl}-2$. However, pretreatment of DC significantly suppressed tBHP-induced depletion of $\mathrm{Bcl}-2$, albeit not concentration-dependent (Fig. 4).

\section{DC suppresses tBHP-induced degradation of IKB and nuclear translocation of NF-kB}

In an effort to examine a possible underlying mechanism by which DC exerts neuroprotection in SH-SY5Y cells, the level of nuclear translocation of NF-kB was examined given the fact that nuclear NF-kB is essential for its proapoptotic roles in various cell culture models (Bian et al., 2001; Wang et al., 2002). In the present study, oxidative stress with tBHP resulted in a significantly decreased intracellular level of IKB in SH-SY5Y cells (Fig. 5). However, pretreatment of DC exhibited a significant attenuation of tHBP-induced degradation of IKB (Fig. 5). To further determine whether the change in the level of IKB affects the nuclear translocation of NF-kB, the level of nuclear NF-kB was examined using confocal microscopy. Immunostaining of p65 subunit of NF-kB was present predominantly in the cytoplasm in basal condition (Fig. 6). tBHP stimulation resulted in apparent nuclear translocation of p65 subunit of NF-kB in SH-SY5Y cells. However, pretreatment of DC appeared to attenuate the tBHP-induced nuclear translocation of p65 subunit of NF-kB in a concentration-dependent manner (Fig. 6).

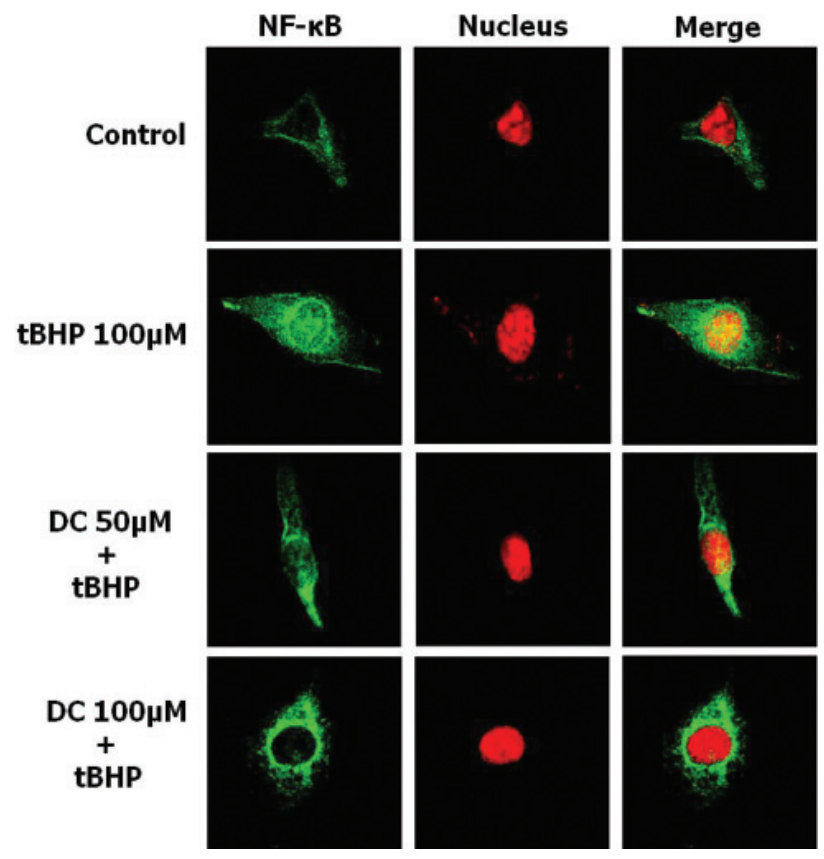

Fig. 6. Blockade of nuclear translocation of p65 subunit of NFkB by DC in tBHP-induced SH-SY5Y human neuroblastoma cells. Localization of NF-kB p65 subunit was determined using a p65 antibody and an Alexa 546-labeled goat anti-rabbit IgG antibody. Nuclei were visualized by propidium iodide (PI) staining. In basal condition, immunostaining of p65 subunit was diffuse throughout the cytoplasm. However, tBHP stimulation resulted in the apparent translocation of p65 subunit into the nucleus. Pretreatment of DC appear to attenuate tBHP-induced nuclear translocation of p65 subunit.

\section{DISCUSSION}

Reactive oxygen species (ROS) may disturb the inherent cellular antioxidant defense system, resulting in damage to biological macromolecules such as nucleic acids, proteins, carbohydrates, and lipids. Such forms of damage have been implicated in several neurodegenerative disorders including Alzheimer's disease (AD), Parkinson's disease (PD), Huntington's disease (HD), and ischemia (Hyslop et al., 1995; Chandra et al., 2000). Therefore, the protection of neuronal cells from reactive oxygen species can be beneficial for the prevention and treatment of these diseases. In the present study, we demonstrated that DC isolated from the stem bark of Rhus verniciflua possesses neuroprotective activity in tBHP-induced neuronal cell death in SH-SY5Y human neuroblastoma cells. DC significantly suppressed tBHP-induced apoptosis and $\mathrm{Bcl}-2$ depletion. Furthermore, DC significantly attenuated tBHP-induced IKB degradation and subsequent nuclear translocation of NF-kB.

Numerous ester derivatives of caffeic acid such as CAPE, chlorogenic acid, pedicularioside $A$, forsythoside $B$, and echinachoside have been reported to exhibit biological activities such as antioxidant and anti-inflammatory properties, inhibition of caspase-3, and maintenance of mitochondrial function (Deng et al., 2004; Jiang et al., 2010; Lapchak, 2007; Li et al., 2008; Wei et al., 2008). Although ester derivatives of caffeic acid with high molecular weight aliphatic alcohol such as DC and 1-eicosanoyl cafferate have been isolated from sophora 
subprostrata (Komatsu et al., 1970) and Echinosophora koreensis (Kang and Kim, 1987), respectively, their biological activities have not been examined. It is presumed that high molecular fatty alcohols in their chemical structures may provide the compounds with lipid solubility, facilitating intracellular penetration through the plasma membrane. In the present study, DC exhibited a significant neuroprotective activity in tBHP-induced neuronal cell death in a micromolar range, suggesting that DC might efficiently enter the cells due to its high lipid solubility. Although DC was not a novel compound, the present study was the first demonstration that DC possesses neuroprotective activity in an oxidative stress-induced neuronal cell death model.

The inappropriate regulation of NF-kB and its downstream genes has been associated with various pathological conditions (Karin et al., 2001; Li and Verma, 2002). Aberrant NF-kB activation was observed in CA1 hippocampal neurons following transient global forebrain ischemia in rats (Clemens et al., 1997; Cho et al., 2008). In addition, it has been reported that NF-kB plays a pro-appototic role in doxorubicin-induced apoptosis (Wang et al., 2002; Ashikawa et al., 2004). In accordance with these reports, the present study showed that pretreatment of DC significantly attenuated tBHP-induced nuclear translocation of NF-kB. However, it has been also suggest that NF-kB might be anti-apoptotic (Romano et al., 2004) and that activation of NF-kB can provide neuroprotection against amyloid $\beta$-induced toxicity (Barger et al., 1995) and oxidative stress (Goodman and Mattson, 1996; Mattson et al., 1997), suggesting a diverse function of NF-kB in the nervous system depending on the cellular context.

In conclusion, the present study demonstrates that DC exerts the neuroprotective activity by inhibiting nuclear translocation of NF-kB against tBHP-induced oxidative stress in SH-SY5Y human neuroblastoma cells. The present study suggests that DC might be a valuable therapeutic agent in the treatment of neuronal cell death-related brain pathologies such as neurodegenerative diseases.

\section{ACKNOWLEDGMENTS}

This work was supported by the Korea Research Foundation Grant funded by the Korean Government (MOEHRD, Basic Research Promotion Fund) (KRF-2008-E00028).

\section{REFERENCES}

Amoroso, S., Gioielli, A., Cataldi, M., Di Renzo, G. and Annunziato, L. (1999) In the neuronal cell line SH-SY5Y, oxidative stress-induced free radical overproduction causes cell death without any participation of intracellular $\mathrm{Ca}^{2+}$ increase. Biochim. Biophys. Acta. 1452, 151-160.

Ashikawa, K., Shishodia, S., Fokt, I., Priebe, W. and Aggarwal, B. B. (2004) Evidence that activation of nuclear factor-kappaB is essential for the cytotoxic effects of doxorubicin and its analogues. Biochem. Pharmacol. 67, 353-364.

Barger, S. W., Horster, D., Furukawa, K., Goodman, Y., Krieglstein, J. and Mattson, M. P. (1995) Tumor necrosis factors alpha and beta protect neurons against amyloid beta-peptide toxicity: evidence for involvement of a kappa B-binding factor and attenuation of peroxide and $\mathrm{Ca}^{2+}$ accumulation. Proc. Natl. Acad. Sci. U S A 92, 93289332.

Bian, X., McAllister-Lucas, L. M., Shao, F., Schumacher, K. R., Feng,
Z., Porter, A. G., Castle, V. P. and Opipari, A. W., Jr. (2001) NF-kappa $B$ activation mediates doxorubicin-induced cell death in N-type neuroblastoma cells. J. Biol. Chem. 276, 48921-48929.

Byun, J. S., Han, Y. H., Hong, S. J., Hwang, S. M., Kwon, Y. S., Lee, H. J., Kim, S. S., Kim, M. J. and Chun, W. (2010) Bark constituents from mushroom-detoxified Rhus verniciflua suppress kainic acid-induced neuronal cell death in mouse hippocampus. Korean J. Physiol. Pharmacol. 14, 279-283.

Chandra, J., Samali, A. and Orrenius, S. (2000) Triggering and modulation of apoptosis by oxidative stress. Free Radic. Biol. Med. 29, 323-333.

Cho, I. H., Hong, J., Suh, E. C., Kim, J. H., Lee, H., Lee, J. E., Lee, S., Kim, C. H., Kim, D. W., Jo, E. K., Lee, K. E., Karin, M. and Lee, S. J. (2008) Role of microglial IKKbeta in kainic acid-induced hippocampal neuronal cell death. Brain 131, 3019-3033.

Clemens, J. A., Stephenson, D. T., Smalstig, E. B., Dixon, E. P. and Little, S. P. (1997) Global ischemia activates nuclear factor-kappa B in forebrain neurons of rats. Stroke 28, 1073-1080; discussion 1080-1071.

Deng, M., Zhao, J. Y., Tu, P. F., Jiang, Y., Li, Z. B. and Wang, Y. H. (2004) Echinacoside rescues the SHSY5Y neuronal cells from TNFalpha-induced apoptosis. Eur. J. Pharmacol. 505, 11-18.

Friedlander, R. M. (2003) Apoptosis and caspases in neurodegenerative diseases. N. Engl. J. Med. 348, 1365-1375.

Goodman, Y. and Mattson, M. P. (1996) Ceramide protects hippocampal neurons against excitotoxic and oxidative insults, and amyloid beta-peptide toxicity. J. Neurochem. 66, 869-872.

Halliwell, B. (1989) Free radicals, reactive oxygen species and human disease: a critical evaluation with special reference to atherosclerosis. Br. J. Exp. Pathol. 70, 737-757.

Hyslop, P. A., Zhang, Z., Pearson, D. V. and Phebus, L. A. (1995) Measurement of striatal $\mathrm{H} 2 \mathrm{O} 2$ by microdialysis following global forebrain ischemia and reperfusion in the rat: correlation with the cytotoxic potential of $\mathrm{H} 2 \mathrm{O} 2$ in vitro. Brain Res. 671, 181-186.

linuma, M., Tanaka, T., Mizuno, M. and Lang, F. A. (1992) Two flavoanones from roots of Sophora leachiana. Phytochemistry 31, 721-723.

Jeon, W. K., Lee, J. H., Kim, H. K., Lee, A. Y., Lee, S. O., Kim, Y. S., Ryu, S. Y., Kim, S. Y., Lee, Y. J. and Ko, B. S. (2006) Anti-platelet effects of bioactive compounds isolated from the bark of Rhus verniciflua Stokes. J. Ethnopharmacol. 106, 62-69.

Jeong, J. S. and Park, J. W. (2008) Carcinostatic effect of allergen removed Rhus verniciflua stokes based traditional Korean medicine on a patient with lung adenocarcinoma; single case report. OPEM 7, 573-578

Jiang, W. L., Tian, J. W., Fu, F. H., Zhu, H. B. and Hou, J. (2010) Neuroprotective efficacy and therapeutic window of Forsythoside $B$ : in a rat model of cerebral ischemia and reperfusion injury. Eur. J. Pharmacol. 640, 75-81.

Kang, S. and Kim, C. (1987) Studies on the Korean Indigenous Plants. Isolation of 1-eicosanoyl cafferate from Echinosophora koreensis. Arch. Pharm. Res. 10, 67-68.

Karin, M., Takahashi, T., Kapahi, P., Delhase, M., Chen, Y., Makris, C., Rothwarf, D., Baud, V., Natoli, G., Guido, F. and Li, N. (2001) Oxidative stress and gene expression: the AP-1 and NF-kappaB connections. Biofactors 15, 87-89.

Kim, M. and Choi, W. (2002) Anticancer and antioxidant activity of allergen-removed extract in Rhus verniciflua stokes. Korean J. Med. Crop Sci 10, 288-293.

Kim, M., Choi, Y. H., Kim W. G. and Kwak S. S. (1997) Antioxidative activity of urushiol derivatives from the sap of lacquer tree (Rhus verniciflua Stokes). Korean J. Plant. Res. 10, 227-230.

Kim, S. R. and Kim, Y. C. (2000) Neuroprotective phenylpropanoid esters of rhamnose isolated from roots of Scrophularia buergeriana. Phytochemistry 54, 503-509.

Kim, Y. (2010) Neuroprotective phenolics in medicinal plants. Arch. Pharm. Res. 33, 1611-1632.

Komatsu, M., Tomimori, T., Hatayama, K. and Makiguchi, Y. (1970) Studies on the constituents of Sophora species. 3. Constituents of the root of Sophora subprostrata Chun et T. Chen. Yakugaku Zasshi 90, 459-462.

Lapchak, P. A. (2007) The phenylpropanoid micronutrient chlorogenic 
acid improves clinical rating scores in rabbits following multiple infarct ischemic strokes: synergism with tissue plasminogen activator. Exp. Neurol. 205, 407-413.

$\mathrm{Li}, \mathrm{Q}$. and Verma, I. M. (2002) NF-kappaB regulation in the immune system. Nat. Rev. Immunol. 2, 725-734.

Li, Y. Y., Lu, J. H., Li, Q., Zhao, Y. Y. and Pu, X. P. (2008) Pedicularioside $A$ from Buddleia lindleyana inhibits cell death induced by 1-methyl-4-phenylpyridinium ions (MPP+) in primary cultures of rat mesencephalic neurons. Eur. J. Pharmacol. 579, 134-140.

Liu, B., Gao, H. M., Wang, J. Y., Jeohn, G. H., Cooper, C. L. and Hong, J. S. (2002) Role of nitric oxide in inflammation-mediated neurodegeneration. Ann. N Y Acad. Sci. 962, 318-331.

Liu, H., Miller, E., van de Water, B. and Stevens, J. L. (1998) Endoplasmic reticulum stress proteins block oxidant-induced $\mathrm{Ca}^{2+}$ increases and cell death. J. Biol. Chem. 273, 12858-12862.

Mattson, M. P., Goodman, Y., Luo, H., Fu, W. and Furukawa, K. (1997) Activation of NF-kappaB protects hippocampal neurons against oxidative stress-induced apoptosis: evidence for induction of manganese superoxide dismutase and suppression of peroxynitrite production and protein tyrosine nitration. J. Neurosci. Res. 49 , 681-697.

Nohl, H., Gille, L., Schonheit, K. and Liu, Y. (1996) Conditions allowing redox-cycling ubisemiquinone in mitochondria to establish a direct redox couple with molecular oxygen. Free Radic. Biol. Med. 20 207-213.

Park, K. Y., Jung, G. O., Lee, K. T., Choi, J., Choi, M. Y., Kim, G. T., Jung, H. J. and Park, H. J. (2004) Antimutagenic activity of flavo- noids from the heartwood of Rhus verniciflua. J. Ethnopharmacol. 90, 73-79.

Romano, M. F., Avellino, R., Petrella, A., Bisogni, R., Romano, S. and Venuta, S. (2004) Rapamycin inhibits doxorubicin-induced NF-kap$\mathrm{paB} / \mathrm{Rel}$ nuclear activity and enhances the apoptosis of melanoma cells. Eur. J. Cancer 40, 2829-2836.

Simonian, N. A. and Coyle, J. T. (1996) Oxidative stress in neurodegenerative diseases. Annu. Rev. Pharmacol. Toxicol. 36, 83-106.

Sul, D., Kim, H. S., Lee, D., Joo, S. S., Hwang, K. W. and Park, S. Y. (2009) Protective effect of caffeic acid against beta-amyloidinduced neurotoxicity by the inhibition of calcium influx and tau phosphorylation. Life Sci. 84, 257-262.

Tsai, S. K., Lin, M. J., Liao, P. H., Yang, C. Y., Lin, S. M., Liu, S. M., Lin, R. H., Chih, C. L. and Huang, S. S. (2006) Caffeic acid phenethyl ester ameliorates cerebral infarction in rats subjected to focal cerebral ischemia. Life Sci. 78, 2758-2762.

Wang, S., Kotamraju, S., Konorev, E., Kalivendi, S., Joseph, J. and Kalyanaraman, B. (2002) Activation of nuclear factor-kappaB during doxorubicin-induced apoptosis in endothelial cells and myocytes is pro-apoptotic: the role of hydrogen peroxide. Biochem. $J$. 367, 729-740.

Wei, X., Ma, Z., Fontanilla, C. V., Zhao, L., Xu, Z. C., Taggliabraci, V., Johnstone, B. H., Dodel, R. C., Farlow, M. R. and Du, Y. (2008) Caffeic acid phenethyl ester prevents cerebellar granule neurons (CGNs) against glutamate-induced neurotoxicity. Neuroscience 155, 1098-1105. 